\title{
Karyotypic diversities in animals and their possible
} causes

Keywords: karyotypes, mammals, coleoptera, evolution, possible causes

\section{Introduction}

All eukaryotic organisms have a species specific set of chromosomes which can be ordered according with their sizes and shapes to make the karyotype. The chromosome sets of animals are highly variable in number and configuration, they range from $2 n=2$ in the Australian ant Myrmecia pilosula to $2 \mathrm{n}=446$ chromosomes in the Morocco butterfly Lysandra atlantica. ${ }^{2}$ Although the levels of karyotypic analyses are from the basic ones of $\alpha$ - and $\beta$-karyology, dealing with number, morphology, and types of sex-chromosome systems, to those of the highest differential resolution of "chromosome paintings" named $\theta$-karyology, most cytogenetic findings in animals are on the former karyologies. ${ }^{2}$ In the present review, we should remind that when we try to get an insight either on chromosomal phylogeny or on cytotaxonomy of a particular group of related species, we should not usually consider the changes due to population polymorphisms, presence of supernumerary B-chromosomes and of chromosomal abnormalities, because they could not provide worth data for distinguishing between species.

Bengston $^{3}$ suggested a method for measuring rates of karyotype evolution in genera of mammals belonging to the same family by the standard deviations (SD) of their species chromosome numbers, which gave two groups depending on whether the animals in the genera had high or low rates of karyotype evolution. In general, mammals of large size and reduced taxonomic diversity yielded low rates whereas those of small size and high taxonomic diversity yielded high rates of karyotype evolution. As found by Bush et al., ${ }^{4}$ the high capacity for dispersal and the absence of a social structure gives large effective population sizes does not promote karyological diversity while low capacity for dispersal (sedentary habits) and/or existence of a social structure in familial groups, gives small effective population sizes where a new chromosomal mutation has a higher chance for fixation in homozygousity by inbreeding or genetic drift. In this sense, Arnason $^{5}$ found also that large mammals, such as cetaceans (whales and dolphins) and pinnipedans (seals and walrus), share a remarkable stability of their karyotypes contrary to that shown in small mammals of the orders Insectivora (shrews and moles) and Rodentia (rats, mouses, squirrels and others). Nevertheless, some large or mediumsized mammals namely those of the genus Equus (horses, donkeys and zebras) and Canidae (dogs, wolves and foxes) display also a great karyotype heterogeneity. ${ }^{6}$

We have also measured the karyological diversity within families and some genera of Coleoptera (beetles) insects to obtain a similar information and trying to reach the possible causative explanations as those found in mammals. ${ }^{7,8}$ The rates of karyological evolution in some representative groups of mammals and beetles based on the standard deviations (SD) of their diploid chromosome numbers are given in Table 1 . The mammals of high dispersal capacity such as cetaceans (whales and dolphins), pinnipedians (seals), and felids (cats) have or have had until recent times large local populations, therefore their
Volume 6 Issue 5 - 2018

\author{
Eduard Petitpierre \\ Department of Biology, University of Balearic Islands, Spain
}

Correspondence: Eduard Petitpierre, Deptartment of Biology, University of Balearic Islands, 07 22 Palma de Mallorca, Spain, Email eduard.petitpierre@uib.es

Received: October 04, 2018 | Published: October 26, 2018

large deme sizes make difficult to fix any new chromosomal mutation and consequently they show low rates of karyological evolution $(\mathrm{SD} \leq 2.0)$. Conversely, many species of Canidae (foxes) are sedentary and display very low vagilities, and the Equidae (horse family) although of high vagility, have social and reproductive structures in herds composed of only one dominant male and several females which both make easier fixing new chromosomal mutations. ${ }^{9,10}$ As expected, these two families Canidae and Equidae have high rates of karyological evolution $(\mathrm{SD} \geq 4.0$ ). Moreover, it is remarkable that all the 11 studied species of the genus Canis (wolves and dogs) share $2 n=78$ chromosomes while seven species of the genus Vulpes (foxes) have a broad range of diploid numbers from $2 n=34$ to $2 n=60$, which can be attributed to their very distinctive vagilities of the former with regard to the latter.

Table I Rates of karyological evolution of diploid numbers in some representative groups of mammals and beetles

\begin{tabular}{|c|c|c|c|c|}
\hline Mammals & Spp. & Range & SD & References \\
\hline Cetacea (whales) & 17 & $42-44$ & 0.64 & 1 \\
\hline Pinnipeda (seals) & 18 & $32-36$ & 1.67 & 1 \\
\hline Felidae (cats) & 30 & $36-38$ & 0.75 & 2,3 \\
\hline $\begin{array}{l}\text { Canidae (wolves, dogs \& } \\
\text { foxes) }\end{array}$ & 29 & $34-78$ & 15.22 & $2-4$ \\
\hline $\begin{array}{l}\text { Equidae (horses, donkeys \& } \\
\text { zebras) }\end{array}$ & 7 & $32-66$ & 4.38 & 2 \\
\hline \multicolumn{5}{|l|}{ Coleoptera } \\
\hline Carabidae & 564 & $8-69$ & 7.58 & 5 \\
\hline Scarabaeidae & 240 & $12-30$ & 1.6 & 5 \\
\hline Coccinellidae & 172 & $12-25$ & 2.68 & 5 \\
\hline Cerambycidae & 157 & $10-36$ & 3.5 & 5 \\
\hline Chrysomelidae & 706 & $8-64$ & 8.26 & 5 \\
\hline Curculionidae & 538 & $12-54$ & 6.93 & 5 \\
\hline
\end{tabular}

In the particular case of Coleoptera (beetles), we have considered two features that have presumably a direct impact on effective deme sizes, the capacity for dispersal apprised in the difference between flying and flightless species, and the trophic selection, on a broad range of vegetal, animal or saprophytic resources, that is polyphagy, 
vs. a narrow range of alimentary preferences, or oligophagy, more rarely monophagy. The flying capacity and/or the polyphagous behavior are aspects likely determining larger deme sizes than the flightless character and/or oligophagous behavior, because the beetles having these latter features would be more sedentary than the former. Although the beetle families cannot be closely classified in one or another of the two pairs of groups, a very rough estimation is possible. Thus, many Carabidae (ground beetles) and at least a fair number of Chrysomelidae (leaf beetles) and Curculionidae (weevils) are flightless whereas most Scarabaeidae (dung beetles and chafers), Coccinellidae (ladybird beetles) and Cerambycidae (longhorn beetles) are flying species. Furthermore, oligophagy or less often monophagy is common in Chrysomelidae and Curculionidae, while polyphagy seems to be most frequent in species belonging to the three other families. In Table 1 we can find a rough correspondence between these features and the rates of karyological evolution: low rates of $\mathrm{SD} \leq 4.0$ in Scarabaeidae, Coccinellidae and Cerambycidae, and high rates of $\mathrm{SD} \geq 4.0$ in Carabidae, Chrysomelidae and Curculionidae. However, within each beetle family there are exceptions to the above features of dispersal capacity and trophic affiliation, and for this reason a more reliable approach to this subject is by using genera instead of families. In this way, we worked out the SDs of diploid chromosome numbers in 24 beetle genera of six families, where a minimum of 15 species per genus have been analyzed, to perform two analyses of variance. ${ }^{7}$ In one of them, the flightless species genera were more variable karyologically than the flying ones but the difference was not significant $(\mathrm{P}=0.18)$, on the contrary, in the second the oligophagous or monophagous $v s$. polyphagous affiliation gave a significant difference $(\mathrm{P}=0.02<0.05)$. It should be pointed out that when species of any beetle genus are flyiers and polyphagous, these two features probably favouring large deme sizes, they never display a high rate of chromosomal evolution.

Finally, we cannot assume that these chromosome mutations dealt with in mammals and beetles have been responsible for processes of speciation, because as King ${ }^{11}$ emphasized, only those mutations that affect the meiotic system, by producing unbalanced and inviable zygots or infertile hybrids, could be invoked for them after the necessary experimental findings.

\section{Acknowledgements}

None.

\section{Conflict of interest}

Author declare that there is no conflict of interest.

\section{References}

1. Crosland MW, Crozier RH. Mymecia pilosula, an ant with only one pair of chromosomes. Science. 1986;231(4743):1278.

2. White MJD. Modes of speciation. W.H. Freeman and Co., San Francisco; 1978.

3. Bengtsson BO. Rates of karyotype evolution in placental mammals. Hereditas. 1980;92(1):37-47.

4. Bush GL, Case SM, Wilson AC, et al. Rapid speciation and chromosomal evolution in mammals. Proc Natl Acad Sci U S A. 1977;74(9):3942-3946.

5. Arnason U. The role of chromosomal rearrangement in mammalian speciation with special reference to Cetacea and Pinnipeda. Hereditas. 1972;70(1):113-118.

6. Matthey R. The chromosome formulae of eutherian mammals. In: Chiarelli AB Capanna E, editors. Cytotaxonomy and vertebrate evolution. London \& New York: Academic Press; 1973. p. 531-615.

7. Petitpierre E. Why beetles have strikingly different rates of chromosomal evolution? Elytron. Bulletin of the European Association of Coleopterology. 1987;1:25-32.

8. Wayne RK. Molecular evolution of the dog family. Trends Genet. 1993;9(6):218-224.

9. Wurster-Hill DH, Centerwall WR. The interrelationships of chromosome banding patterns in canids, mustelids, hyena, and felids. Cytogenet Cell Genet. 1982;34(1-2):178-192.

10. John B, Miklos GLG. The eukaryote genome in development and evolution. London: Allen \& Unwin;1988.

11. King M. Species evolution the role of chromosome change. UK: Cambridge Univ Press; 1993. 